\title{
VOLATILIDAD DE LOS PRECIOS DE PRODUCTOS AGRÍCOLAS ECUATORIANOS, BANANO, CACAO VERSUS LOS DE PRIMERA NECESIDAD: MAÍZ, SOJA, TRIGO Y ARROZ. DISEÑO DE UN ESTUDIO EXPLORATORIO PARA EL PERIODO DESDE 1991 - 2009
}

\author{
PRICE VOLATILITY OF ECUADORIAN AGRICULTURAL PRODUCTS, BANANAS, \\ COCOA VERSUS OF PRIMARY NECESSITY: CORN, SOYBEAN, WHEAT AND RICE. \\ DESIGN OF AN EXPLORATORY STUDY FOR THE PERIOD FROM 1991 - 2009
}

\section{FREDDY CAMACHO VILLAGÓMEZ2', CHRISTIAN BERMÚDEZ GALLEGOS², YANINA BAJAÑA VILLAGÓMEZ ${ }^{3}$}

\footnotetext{
1 Universidad Católica de Santiago de Guayaquil.freddy.camacho@cu.ucsg.edu.ec

2 Universidad Católica de Santiago de Guayaquil. christian.bermudez@cu.ucsg.edu.ec

3 Universidad Católica de Santiago de Guayaquil. yanina.bajana@cu.ucsg.edu.ec
}

RESUMEN

El objetivo de la presente investigación es determinar la volatilidad de los precios internacionales de los productos agrícolas ecuatorianos o también llamados suntuarios versus los de primera necesidad. En la actualidad, los organismos de control desconocen con claridad el impacto de los cambios de los precios debido a diferentes factores como son el aumento del consumo de los alimentos, recesiones y factores externos que afectan directamente 0 indirectamente a la producción y consecuentemente a los precios. Se utilizó el método cuantitativo con un enfoque de tipo exploratorio y descriptivo. Los productos tomados para el estudio fueron el cacao, banano versus el maíz, soja, trigo y arroz. Se realiza un análisis de forma empírica, recopilando información secundaria de bases de datos de fuentes oficiales. Entre los resultados obtenidos, destaca que existe una alta volatilidad de los precios de los productos ecuatorianos versus los de primera necesidad en el contexto internacional. Esto permite concluir, que para los productores ecuatorianos es muy riesgoso ya que puede influir en el cambio de los precios.

PALABRAS CLAVE: volatilidad, producción, precios, consumo, deflactor.
ABSTRACT

The objective of this research is to determine the volatility of international prices of Ecuadorian agricultural products or so-called sumptuary versus necessities. Currently, control agencies know clearly the impact of price changes due to factors such as increased consumption of food, recessions and external factors affecting directly or indirectly to the production and consequently prices. The quantitative method with a focus on exploratory and descriptive research was used for supporting this article. Products taken for the study were cocoa, bananas versus corn, soybean, wheat and rice. Empirical analysis is performed, collecting secondary information databases from official sources. Among the results, they emphasize: there is a high price volatility of Ecuadorian products versus staple in the international context. This leads to the conclusion that for Ecuadorian producers is very risky because it can influence change in prices.

KEYWORDS: volatility, production, prices, consumption deflator. 


\section{INTRODUCCIÓN}

La presente investigación científica permite explicar la volatilidad de los precios en mercados internacionales de los principales productos ecuatorianos de exportación, con respecto a los de primera necesidad.

La investigación se enmarcó en un análisis cuantitativo, es decir, un análisis de tipo marco teórico, enfocándolo de manera descriptiva y lógica deductiva. El estudio está dentro de un horizonte longitudinal. El método utilizado fue la recopilación de datos. Para ello, las fuentes oficiales consultadas fueron: la Food and Agriculture Organization (FAO), la Base de datos sobre comercio de productos de la División de Estadísticas de la Organización de Naciones Unidas (COMTRADE) y el Fondo Monetario Internacional (FMI), cada una de ellas para la construcción de las variables en estudio, y consecuentemente el análisis de los resultados.

Esta investigación es relevante también, para países que como Ecuador, exportan productos agrícolas secundarios o como inicialmente se los denominó suntuarios. Puede ser tomado como base para tomar precauciones referentes a políticas de gobierno con respecto a estos sectores agrícolas, con el fin de proteger a los agricultores en los tiempos de crisis económica bajo el contexto de disminución de los precios internacionales provocados por diferentes factores.

Existe una amplia gama de productos alimenticios que satisfacen las necesidades del ser humano. Según fuentes de FAO, dentro de estos se encuentran productos de primera necesidad como el maíz, trigo, soja y el arroz. Ellos, además, constituyen los productos alimenticios con mayor producción y consumo a nivel mundial. En el contexto ecuatoriano, los principales productos alimenticios de producción y exportación son: cacao, banano y camarón, constituyéndose como los más representativos en la Balanza Comercial.

El estudio realizado comprende desde el año 1991 hasta el 2009. En este período el Ecuador sufrió dos recesiones (1998 y 2008 - 2009), coincidiendo esta última con la recesión mundial. La obtención de datos permitirá realizar un pronóstico.

Es importante señalar que a nivel mundial, el cambio climático, entre otros elementos ha provocado cambios en las economías de los países. Esto ha traído como consecuencia un sinnúmero de recesiones mundiales originando una disminución de los ingresos y estos provocan un decrecimiento en el consumo de las familias, lo cual se ve afectado al Producto Interno Bruto (PIB). Por ello, se hace imperioso realizar un análisis de esta magnitud dado el entorno mundial en el cual nos encontramos involucrados y nos vemos afectados en la globalización.

La política pública sobre la agricultura a nivel mundial pretende fomentar la producción de alimentos ante cambios de la volatilidad de los precios en los mercados nacionales e internacionales. Con esto se busca reactivar la economía de los países, reflejado en el incremento del PIB. Sin embargo, esta situación se acompaña de un aumento de los precios debido a la escasez de alimentos. En este sentido, la presente investigación se vuelve de vital importancia para los tomadores de decisiones públicas en el sector agrícola.

De acuerdo con estos análisis a priori, el beneficio o perjuicio de un país a través de su balanza comercial es la producción y exportación de bienes o servicios para aportar a la balanza comercial de manera positiva y que permita tener una buena imagen a nivel internacional, generando así mayores riquezas.

En la actualidad se debe realizar un análisis profundo sobre la agricultura a nivel mundial dado algunos cambios que se han suscitado en la producción y consumo de alimentos ante variaciones de los precios. Con el transcurso de los años, las variables precio y consumo han venido cambiando ante una explotación excesiva de recursos naturales y la contaminación mundial, que de alguna manera ha comenzado a hacer efecto en estos sectores.

Otra variable que afecta directamente al precio de los productos agrícolas es el constante crecimiento de la población, ya que en los últimos años el crecimiento poblacional ha sido exponencial, y esto se ha visto reflejado en el consumo y consecuentemente en los precios.

De acuerdo a los últimos datos presentados por el Banco Mundial (2014), se estima que el crecimiento poblacional del 2025 al 2030 aumentará en un $0,8 \%$.

Aunque el crecimiento de la población vaya disminuyendo se va haciendo importante definir cuáles son los productos de primera necesidad ante la escases inminente de recursos.

A lo largo de su historia el Ecuador ha mantenido en promedio una balanza comercial negativa, dado que el nivel de importaciones es mayor al nivel de exportaciones. Como con- 
secuencia ha resultado una disminución del PIB, considerando que uno de sus componentes principales constituye el rubro de las exportaciones netas, lo cual evidencia una afectación directa de manera negativa en el PIB.

OBJETIVOS

El objetivo general planteado es determinar la volatilidad de los precios de los productos agrícolas ecuatorianos contra los de primera necesidad de consumo a nivel mundial.

Para alcanzar este objetivo se han establecidos como específicos los siguientes:

1. Determinar la producción del cacao, banano, maíz, soja, trigo y arroz en las bases de fuentes oficiales como la FAO.

2. Explorar la tendencia desde 1991 al 2009 de los precios de los productos analizados.

3. Calcular el índice de precios de los Estados Unidos como un indicador promedio a nivel mundial de la Reserva Federal de Saint Louis de Estados Unidos.

4. Deflactar los precios de los productos.

5. Cálcular la desviación estándar y el promedio de los productos deflactados.

6. Determinar la volatilidad de los precios.

METODOLOGÍA

La presente investigación puede ser enmarcada como de tipo descriptivo y exploratoria, con una lógica deductiva. Busca encontrar los objetivos planteados, con un diseño no experimental. El diseño de la investigación es un estudio longitudinal con fuente de datos secundaria a nivel mundial.

Se tomaron en consideración seis productos, siendo dos de ellos de exportación de Ecuador y los otros cuatro restantes de producción a nivel mundial de orden primario.

\section{ANÁLISIS Y RECOPILACIÓN DE DATOS}

La recopilación de los datos se realizó a partir de fuentes oficiales como son la FAO y la Reserva Federal de Saint Louis de Estados Unidos. Es preciso indicar que todo el estudio se encuentra basado en términos de toneladas para homogeneizar las bases de datos, ya que nos permite simplificar el análisis en una misma unidad de estudio. La investigación abarcó el período 1991-2009.
ETAPA I

En primer lugar, se investigó la producción de productos alimenticios como son el arroz, trigo, soja, maíz, banano y cacao reflejados en la Tabla 1. El mismo que refleja la agrupación de commodities a nivel mundial en términos generales.

tabla 1. PRODUCCIÓN TOTAL DE PRODUCtOS agríCOLAS EN EL PERí́0D 1991-2009

\begin{tabular}{|c|c|c|c|c|c|c|}
\hline & \multicolumn{2}{|c|}{ ECUADOR } & EEUU & CHINA & CHINA & EUU \\
\hline 10 & BANANA & СACAO & MAÍZ & ARROZ & TRIGO & SOJA \\
\hline 991 & 25300 & 100454 & 189866000 & 185692640 & 81 & 54065000 \\
\hline 992 & 3994640 & 93999 & 240719000 & 188291880 & 101591326 & 200 \\
\hline 993 & 22010 & 82729 & 160985000 & 179746930 & 106394921 & 5088600 \\
\hline 994 & 5085920 & 81163 & 255293000 & 177994400 & 99301440 & 5000 \\
\hline 995 & 5403300 & 85505 & 187969000 & 187297970 & 102211429 & 4000 \\
\hline 996 & 5726620 & 93821 & 234527000 & 197032900 & 110569193 & 64782000 \\
\hline 997 & 7494120 & 83385 & 233867000 & 202771840 & 123290085 & 7317700 \\
\hline 998 & 63440 & 35006 & 2000 & 60 & 66 & 745 \\
\hline 1999 & 6392020 & 94687 & 239549000 & 200403300 & 113880088 & 72223000 \\
\hline 000 & 6477040 & 99875 & 251852000 & 189814060 & 99636127 & 75053800 \\
\hline 001 & 6077040 & 76030 & 241375000 & 179304900 & 938 & 78669900 \\
\hline 02 & 5611440 & 87986 & 00 & 0 & 902 & 750 \\
\hline 2003 & 6453810 & 88263 & 256227000 & 162304280 & 86488264 & 66781400 \\
\hline 004 & 6132280 & 89680 & 299874000 & 180522610 & 91952238 & 85013900 \\
\hline 005 & 6118 & 93658 & 282 & 182055140 & 97445196 & 83504900 \\
\hline 006 & 61 & 61 & 267 & 50 & 71 & 869 \\
\hline 007 & 6002300 & 85891 & 331175000 & 187397460 & 109298296 & 72857700 \\
\hline 88 & 67 & 10 & 0 & 30 & 2 & 700 \\
\hline & 20 & 1582 & 0000 & 70 & 364 & 91417300 \\
\hline
\end{tabular}

Fuente: Food and Agriculture Organization (FAO, 2015).

Luego de bajar la base de datos en términos brutos se procedió a llevar la unidad de peso de kilogramos a toneladas de cada uno de los productos. A continuación fue depurada la base únicamente con los seis productos a analizar.

ETAPA II

En la segunda etapa se recopiló la información sobre los principales exportadores. En la Tabla 1 se muestra el volumen total de los productos de Ecuador en relación a los principales productores de primera necesidad. Adicionalmente, cabe recalcar que aunque China sea el principal productor de arroz y trigo no significa que sea el principal exportador de estos productos. Consecuentemente se evidenció que Estados Unidos (EEUU) es el principal exportador de arroz y trigo. El nivel de producción de este país es detallado a continuación en la Tabla 2. 
TABLA 2. PRINCIPALES PAÍSES EXPORTADORES DE ARROZ Y SOJA

\begin{tabular}{|rrr|}
\hline & EEUU & EEUU \\
\hline Año & ARROZ & TRIGO \\
\hline 1991 & 7230000 & 53890000 \\
\hline 1992 & 8149000 & 67136000 \\
\hline 1993 & 7081000 & 65222000 \\
\hline 1994 & 8971100 & 63168000 \\
\hline 1995 & 7887000 & 59404000 \\
\hline 1996 & 7783600 & 61982000 \\
\hline 1997 & 8300700 & 67536000 \\
\hline 1998 & 8364200 & 69327000 \\
\hline 1999 & 9343950 & 62567300 \\
\hline 2000 & 8657820 & 60639400 \\
\hline 2001 & 9764500 & 52999900 \\
\hline 2002 & 9568990 & 43704000 \\
\hline 2003 & 9067180 & 63803300 \\
\hline 2004 & 10539800 & 58697000 \\
\hline 2005 & 10107500 & 57242000 \\
\hline 2006 & 8826230 & 49216000 \\
\hline 2007 & 8998730 & 55820400 \\
\hline 2008 & 9241170 & 68016100 \\
\hline 2009 & 9972230 & 60365700 \\
\hline & & \\
\hline
\end{tabular}

Fuente: Food and Agriculture Organization (FAO, 2015).

ETAPA III

En la tercera etapa de la investigación, en base a la recopilación de la información referente a los precios de los productos del período analizado (FAO, 2015), mostrados en la Tabla 3, se generaron gráficos del comportamiento a través de series de tiempo a lo largo de los años de estudio de los productos analizados.

Se puede observar en la Figura 1, tomada de FAO (2015), que en el período de 1998 al 2005 el precio del banano tuvo una creciente significativa, como resultado de un lapso de recesión originado por la crisis económica de 1998.

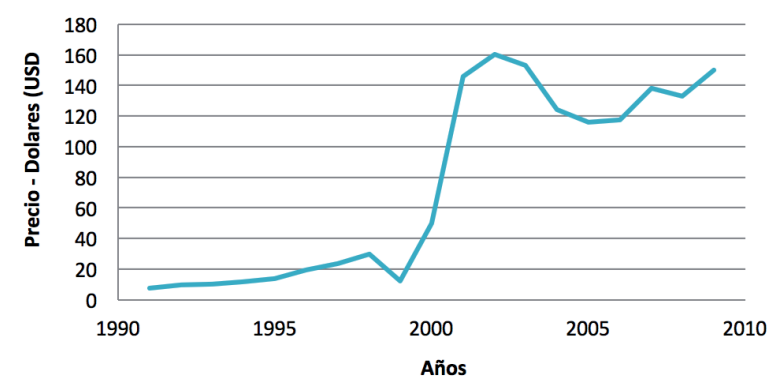

Figura 1: Tendencia del precio del banano, período 1990 al 2009.

En la Figura 2, tomada de FAO (2015), se evidencia que el precio del cacao tiene una tendencia creciente desde los inicios del año 1990. Sin embargo, en el año 2005 cayó su precio, pero seguido de esto recupera su tendencia al alza.

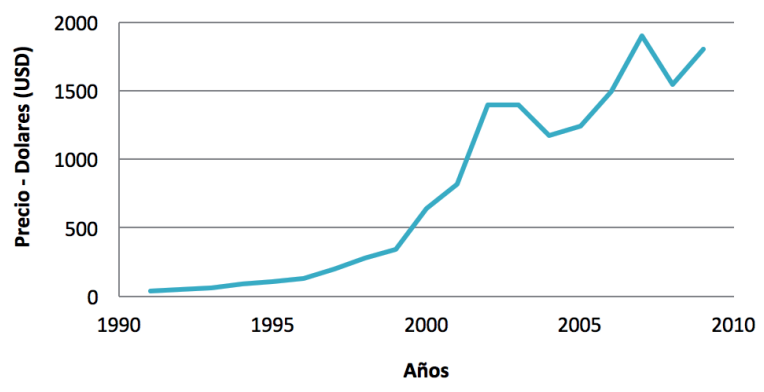

Figura 2. Tendencia del precio del cacao, período 1990 al 2009.

Como se evidencia en la Figura 3, tomada de FAO (2015), el precio del maíz, soja, arroz y trigo guardan un comportamiento uniforme entre estos, a diferencia del cacao y banano mostrada en las figuras anteriores (1 y 2); no reflejan una volatilidad marcada, lo cual será corroborado en el cálculo desarrollado y mostrado en la Tabla 6 .

TABLA 3. PRECIOS CORRIENTES POR PRODUCTO EN EL PERÍODO 1991-2009.

$\begin{array}{lllllllllllllllllllll}\text { PAÍS } & \text { PRODUCTO } & 1991 & 1992 & 1993 & 1994 & 1995 & 1996 & 1997 & 1998 & 1999 & 2000 & 2001 & 2002 & 2003 & 2004 & 2005 & 2006 & 2007 & 2008 & 200\end{array}$

\begin{tabular}{lllllllllllllllllllll}
\multirow{2}{*}{ ECUADOR } & BANANO & 7.7 & 9.7 & 10.4 & 11.7 & 13.6 & 19.3 & 23.4 & 29.6 & 12.4 & 50 & 146 & 160 & 153 & 124 & 116 & 117.3 & 138 & 133.1 & 149.8 \\
& CACAO & 35.7 & 48.2 & 59.3 & 90.5 & 106.3 & 132.1 & 197.8 & 277.9 & 344.4 & 640.3 & 816.5 & 1397.7 & 1397.5 & 1175.2 & 1242.7 & 1491.9 & 1901.6 & 1543.7 & 1803.1 \\
& MAÍZ & 93 & 81 & 98 & 89 & 128 & 140 & 96 & 76 & 72 & 73 & 78 & 91 & 95 & 81 & 79 & 120 & 165 & 160 & 146 \\
EE.UU. & SOJA & 205 & 204 & 235 & 201 & 247 & 247 & 238 & 181 & 170 & 167 & 161 & 203 & 270 & 211 & 208 & 236 & 371 & 366 & 347 \\
& ARROZ & 167 & 130 & 176 & 149 & 202 & 212 & 221 & 196 & 131 & 124 & 94 & 99 & 178 & 162 & 169 & 220 & 282 & 370 & 315 \\
& TRIGO & 107 & 119 & 120 & 127 & 167 & 175 & 124 & 97 & 91 & 96 & 102 & 131 & 125 & 125 & 126 & 157 & 212 & 295 & 195 \\
\hline
\end{tabular}

Fuente: Food and Agriculture Organization (FAO, 2015). 


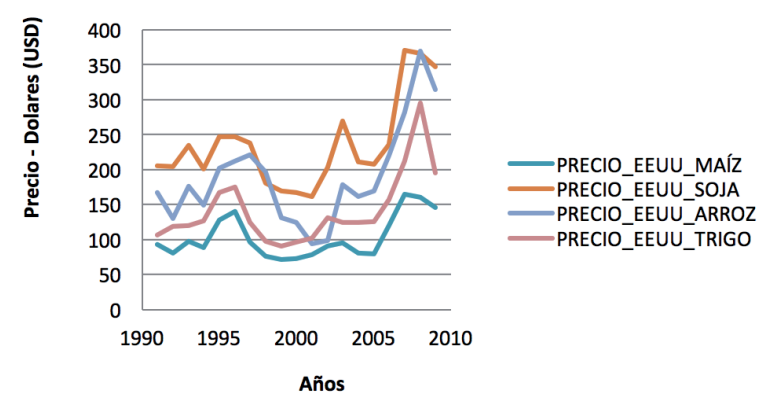

Figura 3. Tendencia de precios de los productos agrícolas del período 1990-2009.

En términos generales, como podemos observar, los seis productos analizados, tanto los primarios como los suntuarios han venido teniendo una tendencia a la alza; siendo más acentuado el crecimiento en los productos ecuatorianos.

ETAPA IV

Como cuarto paso se calculó el índice de precios tomando como referencia el Índice de Precios al Consumidor (IPC) de Estados Unidos. Para ello se bajó la serie de tiempos en la página de la Reserva Federal de Saint Louis, la cual se refleja en la Tabla 4. El índice de precios de los EE.UU. es un indicador promedio a nivel mundial.

\section{TABLA 4 ÍNDICE DE PRECIOS}

\begin{tabular}{|rrrr|}
\hline AÑ̃ & $\begin{array}{r}\text { ÍNDICE DE } \\
\text { PRECIOS }\end{array}$ & AÑ̃ & $\begin{array}{r}\text { INDICE DE } \\
\text { PRECIOS }\end{array}$ \\
\hline 1991 & 136.161739 & 2001 & 177.0400188 \\
\hline 1992 & 140.3024936 & 2002 & 179.8620188 \\
\hline 1993 & 144.4709814 & 2003 & 183.9976458 \\
\hline 1994 & 148.2199002 & 2004 & 188.9000034 \\
\hline 1995 & 152.3795886 & 2005 & 195.2500357 \\
\hline 1996 & 156.8525357 & 2006 & 201.5531321 \\
\hline 1997 & 160.5229614 & 2007 & 207.3309798 \\
\hline 1998 & 163.0062209 & 2008 & 215.2380293 \\
\hline 1999 & 166.5774085 & 2009 & 214.5571084 \\
\hline 2000 & 172.1837957 & & \\
\hline
\end{tabular}

Fuente: Reserva Federal de Saint Louis (2016).

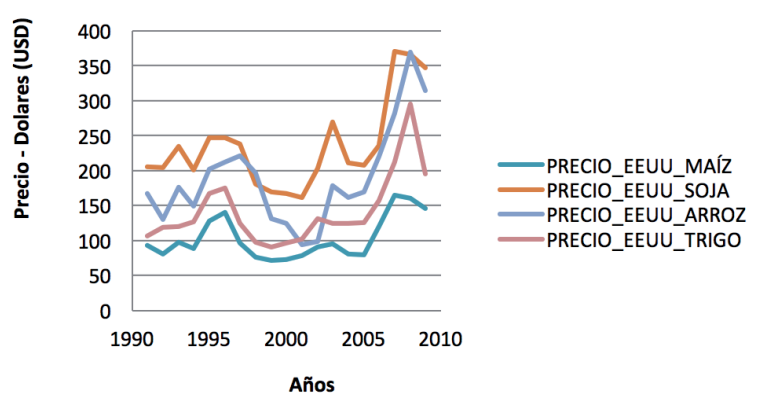

Figura 4. Tendencia de índice de precios de EE.UU.

ETAPA V

Y como quinto paso se deflactaron los precios de todos los productos a analizar. Para ello se dividieron los precios corrientes de los productos para el índice de precios, a continuación son mostrados en la Tabla 5.

TABLA 5 PRECIOS DEFLACTADOS POR PRODUCTOS

\begin{tabular}{|c|c|c|c|}
\hline & ECU & & EE.UU. \\
\hline AÑ̃o & BANANO & CACAO & MAíz \\
\hline 1991 & 0.056550394 & 0.262188191 & 0.683011253 \\
\hline 1992 & 0.069136334 & 0.343543431 & 0.577324023 \\
\hline 1993 & 0.071986775 & 0.410463052 & 0.678336916 \\
\hline 1994 & 0.078936769 & 0.61057928 & 0.600459182 \\
\hline 1995 & 0.089250799 & 0.6975999993 & 0.840007518 \\
\hline 1996 & 0.123045508 & 0.842192314 & 0.892558092 \\
\hline 1997 & 0.145773538 & 123.222 .247 & 0.598045284 \\
\hline 1998 & 0.181588162 & 170.484 .291 & 0.466239875 \\
\hline 1999 & 0.074439866 & 2.067.507.251 & 0.432231481 \\
\hline 2000 & 0.290387372 & 3.718 .700 .691 & 0.423965564 \\
\hline 2001 & 0.824672303 & 4.611 .951 .612 & 0.440578354 \\
\hline 2002 & 0.8895708 & 7.770 .956 .922 & 0.505943393 \\
\hline 2003 & 0.831532378 & 7.595.205.871 & 0.516310954 \\
\hline 2004 & 0.656431963 & 6.221 .280 .989 & 0.428798298 \\
\hline 2005 & 0.594110006 & 6.364.659.526 & 0.404609401 \\
\hline 2006 & 0.581980537 & 7.402.018.439 & 0.595376508 \\
\hline 2007 & 0.665602411 & 9.171 .808 .293 & 0.795828969 \\
\hline 2008 & 0.618385145 & 7.172.059.719 & 0.74336306 \\
\hline 2009 & 0.698182415 & 8.403.823.177 & 0.680471512 \\
\hline 1991 & 1.226 .482 .573 & 0.785830152 & 150.556 .244 \\
\hline 1992 & 0.926569419 & 0.848167392 & 1.454 .001 .243 \\
\hline 1993 & 1.218 .237 .727 & 0.830616632 & 1.626 .624 .237 \\
\hline 1994 & 1.005 .263 .125 & 0.856835012 & 1.356 .093 .209 \\
\hline 1995 & 1.325 .636 .864 & 1.095 .947 .309 & 1.620 .952 .007 \\
\hline 1996 & 1.351 .587 .968 & 1.115 .697 .615 & 1.574.727.491 \\
\hline 1997 & 1.376 .750 .081 & 0.772475158 & 1.482 .653 .933 \\
\hline 1998 & 1.202 .408 .098 & 0.595069314 & 111.038 .707 \\
\hline 1999 & 0.786421167 & 0.546292566 & 1.020 .546 .553 \\
\hline
\end{tabular}




\begin{tabular}{crrr} 
ECUADOR & CACAO & $\begin{array}{r}\text { EE.UU. } \\
\text { MAíz }\end{array}$ \\
AÑo & BANANO & 0.957543755 & 0.969893824 \\
\hline 2000 & 0.720160684 & 0.509 \\
\hline 2001 & 0.5309534 & 0.576140924 & 0.909398909 \\
\hline 2002 & 0.550421933 & 0.728336093 & 1.128 .642 .953 \\
\hline 2003 & 0.967403682 & 0.679356518 & 1.467 .410 .079 \\
\hline 2004 & 0.857596596 & 0.661725769 & 1.116 .993 .098 \\
\hline 2005 & 0.86555682 & 0.645326386 & 1.065 .300 .701 \\
\hline 2006 & 1.091 .523 .598 & 0.778950932 & 1.170 .907 .133 \\
\hline 2007 & 1.360 .144 .057 & 1.022 .519 .646 & 178.940 .938 \\
\hline 2008 & 1.719 .027 .075 & 1.370 .575 .641 & 1.700 .442 .999 \\
\hline 2009 & 1.468 .140 .592 & 0.908848938 & 1.617 .285 .033 \\
\hline
\end{tabular}

Fuente: Datos secundarios de Food and Agriculture Organization (FA0, 2015).

ETAPA VI

Como último paso se determinaron la desviación estándar y el promedio de los precios ya deflactados. Luego se dividió la desviación estándar con el promedio de los precios deflactados de cada producto. Este resultado muestra la volatilidad que se observa en la Tabla 6 .

TABLA 6. VOLATILIDAD DE LOS PRECIOS

\begin{tabular}{llrrr}
\hline PAIS & PRODUCTO & DESVIACIÓN & PROMEDIO & VOLATILIDAD \\
\hline \multirow{2}{*}{ ECUADOR } & BANANO & 0.315478447 & 0.396924393 & 0.794807404 \\
& CACAO & 3.292 .559 .268 & 4.031 .768 .639 & 0.816653822 \\
EE.UU. & MAÍZ & 0.15044887 & 0.594918928 & 0.252889701 \\
& ARROZ & 0.320599628 & 1.081 .593 .972 & 0.29641403 \\
& TRIGO & 0.218380372 & 0.809276618 & 0.269846387 \\
& SOJA & 0.276397052 & 1.351 .959 .594 & 0.204441799 \\
\hline
\end{tabular}

Fuente: Datos secundarios de Food and Agriculture Organization (FA0, 2015).
RESULTADOS

Como resultado final de este análisis podemos observar que la volatilidad de los precios de los productos ecuatorianos, banano y cacao es muy alta con respecto a los productos de primera necesidad que son más estables. Por lo tanto, se vuelve necesario realizar una investigación más profunda, que determine cuáles son los factores que producen la volatilidad de los precios tan elevada para los productos ecuatorianos en el contexto externo. Luego, observar cómo estos afectan a los productores de países en desarrollo.

Dados los resultados se puede inferir que el precio de los productos agrícolas ecuatorianos es muy volátil. Esto puede darse debido a ciertas circunstancias: (a) el cambio climático que provoca un sinnúmero de pérdidas económicas para los agricultores.

En la época invernal las fuertes lluvias provocan escasez; y (b) las recesiones originadas a nivel mundial, son un factor muy correlacionado con el sector agrícola.

REFERENCIAS BIBLIOGRÁFICAS

Banco Mundial. (2014). Indicadores del desarrollo mundial. Recuperado de http://www.bancomundial.org/

FAO. (2015). Agricultura mundial. Recuperado de http://www.fao.org/news/archive/news-bydate/2015/es/

Reserva Federal de Saint Louis. (2016). Consumer Price Index for All Urban Consumers. Recuperado de https://research.stlouisfed.org/fred2/ series/CPIAUCSL. 